\title{
Regulation of Eukaryotic Initiation Factor 4E by Converging Signaling Pathways during Metabotropic Glutamate Receptor-Dependent Long-Term Depression
}

\author{
Jessica L. Banko, ${ }^{1 \star}$ Lingfei Hou, ${ }^{1 *}$ Francis Poulin, ${ }^{3}$ Nahum Sonenberg, ${ }^{3}$ and Eric Klann ${ }^{1,2}$ \\ Departments of ${ }^{1}$ Molecular Physiology and Biophysics and ${ }^{2}$ Neuroscience, Baylor College of Medicine, Houston, Texas 77030, and ${ }^{3}$ Department of \\ Biochemistry, McGill University, Montreal, Quebec, Canada H3G 1Y6
}

\begin{abstract}
Long-term depression (LTD) is an activity-dependent decrease in synaptic efficacy that can be induced in hippocampal area CA1 by pharmacological application of the selective group I metabotropic glutamate receptor (mGluR) agonist 3,5-diyhroxyphenylglycine (DHPG). Recent work has demonstrated that DHPG-induced LTD recruits at least two signal transduction pathways known to couple to translation, the mitogen-activated protein kinase kinase (MEK)- extracellular signal-regulated kinase (ERK) signaling pathway and the phosphoinositide 3-kinase (PI3K)-Akt-mammalian target of rapamycin (mTOR) signaling pathway. However, it remains unclear which translation factors are engaged by these two signaling pathways during mGluR-LTD. In this study, we investigated whether the group I mGluRs couple to the cap-dependent translation proteins: Mnk1, eIF4E, and 4E-BP. We found that both the MEK-ERK and PI3K-mTOR signaling pathways are critical for the DHPG-induced regulation of these translation factors. Furthermore, we demonstrate that increasing eIF4F complex availability via the genetic elimination of 4E-BP2 can enhance the degree of LTD achieved by DHPG application in an ERK-dependent manner. Our results provide direct evidence that cap-dependent translation is engaged during mGluR-LTD and demonstrate that the MEK-ERK and PI3K-mTOR signaling pathways converge to regulate eIF4E activity after induction of DHPG-LTD.
\end{abstract}

Key words: protein synthesis; hippocampus; mGluR; ERK; synaptic plasticity; PI3 kinase

\section{Introduction}

The cellular mechanisms underlying learning and memory are generally attributed to long-lasting changes in synaptic strength. Long-term depression (LTD) is an activity-dependent decrease in synaptic strength that can be mediated by two distinct glutamate receptor classes in the hippocampus (Oliet et al., 1997), one dependent on the activation of NMDA receptors (Mulkey and Malenka, 1992) and the other dependent on the activation of metabotropic glutamate receptors (mGluRs) (Bolshakov and Siegelbaum, 1994). The group I mGluRs, mGluR1 and mGluR5, are activated selectively by 3,5-dihydroxyphenylglycine (DHPG). Application of DHPG $(5 \mathrm{~min}, 100 \mu \mathrm{M})$ to hippocampal slices reliably induces LTD in the CA1 region of the hippocampus (Palmer et al., 1997; Fitzjohn et al., 1999; Huber et al., 2000) and faithfully engages local protein synthesis (Weiler and Greenough, 1993; Huber et al., 2000; Job and Eberwine, 2001). Growing evidence suggests that this mGluR-induced protein synthesis facilitates morphological changes such as spine elongation (Vanderk-

\footnotetext{
Received Aug. 1, 2005; accepted Jan. 6, 2006.

This work was supported by National Institutes of Health Grants NS034007 and NSO47384 (E.K.) and T32 HL07676 (J.L.B.), the FRAXA Research Foundation (E.K.), a Doctoral Award from the Canadian Institutes for Health Research (CIHR) (F.P.), and grants from the (IHR and the Howard Hughes Medical Institute (HHMI) (N.S.). N.S. is a Distinguished Investigator of the CIHR and an HHMI International Scholar.

*J.L.B. and L.H. contributed equally to this work.

Correspondence should be addressed to Dr. Eric Klann, Department of Molecular Physiology and Biophysics, Baylor College of Medicine, Houston, TX 77030. E-mail: eklann@bcm.tmc.edu.

DOI:10.1523/JNEUROSCI.5196-05.2006

Copyright $\odot 2006$ Society for Neuroscience $\quad$ 0270-6474/06/262167-07\$15.00/0
}

lish and Edelman, 2002) and the regulation of glutamate receptor trafficking (Snyder et al., 2001), both of which likely serve to facilitate the expression of LTD.

Recently, we described a signaling pathway by which group I mGluRs could couple to translation machinery by demonstrating the activation of the phosphoinositide 3-kinase (PI3K)-Aktmammalian target of rapamycin (mTOR) signaling pathway during mGluR-LTD (Hou and Klann, 2004). This pathway has been implicated in the regulation of several translation factors that contribute to protein synthesis. For example, S6 kinase, eukaryotic initiation factor (eIF) 4E-binding protein (4E-BP), and eukaryotic elongation factor 2 (eEF2) kinase each play different roles during distinct phases of protein synthesis and are regulated by mTOR (for review, see Hay and Sonenberg, 2004). However, modulation of translation factors that directly interact with the mRNA during the events of translation have yet to be demonstrated during mGluR-LTD. Here, we describe specific signal transduction events that couple the group I mGluRs to capdependent translation initiation factors during mGluR-LTD.

Cap-dependent mRNA translation commences with the binding of the multiprotein complex eIF4F at the $5^{\prime}$ terminus of the mRNA (Gingras et al., 1999). Components of the eIF4F complex include the cap-binding protein eIF4E, the RNA helicase eIF4A, and the scaffolding protein eIF4G. Inhibition of cap-dependent initiation is achieved via the $4 \mathrm{E}-\mathrm{BPs}$, which compete with eIF4G for binding eIF4E (Pause et al., 1994; Poulin et al., 1998). Of the three known $4 \mathrm{E}-\mathrm{BP}$ isoforms, $4 \mathrm{E}-\mathrm{BP} 2$ is the predominant isoform expressed in 
the hippocampus (Banko et al., 2005). Rapamycin-sensitive hyperphosphorylation of the 4E-BPs disrupts the 4E-BP-eIF4E interaction, allowing formation of the eIF4F complex and translation initiation (Beretta et al., 1996; Lin and Lawrence, 1996; Gingras and Sonenberg, 1997; Gingras et al., 1998). To determine the extent to which activation of cap-dependent translation initiation factors contribute to mGluR-LTD, we also investigated mGluR-LTD in mice that lack the cap-dependent translation repressor protein $4 \mathrm{E}-\mathrm{BP} 2$. The results of this study implicate the coordinated regulation of the eIF4F complex via $4 \mathrm{E}-\mathrm{BP} 2$ to be a critical determinant for the magnitude of LTD achieved after activation of the group I mGluRs.

\section{Materials and Methods}

Materials. Unless noted otherwise, all primary antibodies used were purchased from Cell Signaling Technology (Beverly, MA). The horseradish peroxidase-linked goat anti-rabbit IgG and the anti-extracellular signalregulated kinase (ERK) antibodies were obtained from Promega (Madison, WI). Indocarbocyanine (Cy3)-conjugated AffiniPure goat antirabbit IgG was purchased from Jackson ImmunoResearch (West Grove, PA). Anti-eIF4G1 antibody was obtained from Bethyl Laboratories (Montgomery, TX). DHPG, 2-methyl-6-(phenylethynyl)-pyridine (MPEP), LY367385, SB203580, U0126, and U0124 were obtained from Tocris Cookson (Ellisville, MO). 2-(4-Morpholinyl)-8-phenyl-4 H-1benzopyran-4-one (LY294002) and wortmannin were purchased from Sigma (St. Louis, MO). Rapamycin was obtained from Cell Signaling Technology. I-Block was obtained from Tropix (Bedford, MA), and enhanced chemiluminescence (ECL) Western blotting detection reagents were obtained from Amersham Biosciences (Piscataway, NJ).

Hippocampal slice preparations. Hippocampal slices $(400 \mu \mathrm{m})$ were prepared from 4-week-old male C57BL/6 mice. Slices were placed in saline solution containing (in mM) $124 \mathrm{NaCl}, 4.4 \mathrm{KCl}, 26 \mathrm{NaHCO}_{3}, 10$ D-glucose, 2 $\mathrm{CaCl}_{2}$, and $2 \mathrm{MgCl}_{2}$, gassed with $95 \% \mathrm{O}_{2} / 5 \% \mathrm{CO}_{2}$, $\mathrm{pH} 7.4$, for $1 \mathrm{~h}$ at room temperature, and transferred to $32^{\circ} \mathrm{C}$ artificial CSF (ACSF) containing (in mм) $125 \mathrm{NaCl}, 2.5 \mathrm{KCl}, 1.25 \mathrm{NaH}_{2} \mathrm{PO}_{4}, 25 \mathrm{NaHCO}_{3}, 2 \mathrm{CaCl}_{2}, 1 \mathrm{MgCl}_{2}$, and 25 D-glucose saturated with $95 \% \mathrm{O}_{2} / 5 \% \mathrm{CO}_{2}, \mathrm{pH} 7.4$, for $1 \mathrm{~h}$. Slices were then exposed to different compounds of interest for the indicated times and snap-frozen over dry ice. The CA1 regions were microdissected and homogenized in ice-cold homogenization buffer (HB) containing phosphatase and protease inhibitor mixtures. Synaptoneurosome fractions were prepared as described previously (Johnson et al., 1997) by passing the homogenate through membranes of decreasing pore size (100 to $5 \mu \mathrm{m})$. The final filtrate was centrifuged at $10,000 \times g\left(20 \mathrm{~min}, 4^{\circ} \mathrm{C}\right)$, and the pellet containing the synaptoneurosomes was resuspended in HB. The protein concentration was measured by the method of Bradford (1976) using bovine serum albumin as the standard.

Immunoprecipitation. Homogenates were precleared for $1 \mathrm{~h}$ with $50 \%$ protein A bead slurry and sequentially incubated with anti-eIF4G1 antibody $(10 \mu \mathrm{g})$ and $50 \%$ protein A slurry for $1 \mathrm{~h}$ at room temperature. The samples were centrifuged for $20 \mathrm{~min}$ at $4^{\circ} \mathrm{C}$, and the supernatant was discarded. Immunoprecipitated protein complexes were washed with fresh $\mathrm{HB}$ and eluted from the beads before analysis by quantitative Western blotting.

Quantitative Western blot analysis. Equivalent amounts of protein for each sample were resolved in 10\% SDS-PAGE and transferred to polyvinylidene difluoride (PVDF) membranes. The PVDF membranes were incubated with reversible Ponceau $S$ protein stain to confirm equal loading and transfer. Membranes were then blocked in Tris-buffered saline containing Tween 20 and $0.24 \%$ I-Block and incubated with the phospho-specific antibody of interest [dual-phospho-ERK antibody (1:5000), total ERK antibody (1: 5000), phospho-Mnk1 antibody (1:1000), total Mnk1 antibody (1:500), phospho-eIF4E antibody (1:1000), total eIF4E (1:1000), phospho-4E-BP1 antibody (1:500), or eIF4G1 antibody (1:1000)] for $1 \mathrm{~h}$ at room temperature. The phospho-4E-BP1 antibody has been shown to cross-react with 4E-BP2 (Banko et al., 2005). Incubation with horseradish peroxidase-linked goat anti-rabbit IgG (1:2500 dilution) for $1 \mathrm{~h}$ at room temperature followed. All blots were developed using ECL. The bands of each Western blot were quantified from film exposures in the linear range for each antibody and normalized to the Ponceau S membrane staining with densitometry using a desktop scanner and NIH Image software to determine the amount of immunoreac- tivity. Graph Pad (San Diego, CA) Prism data analysis software was used for graph production and statistical analysis. Data represent mean + SEM. Statistical analysis via Student's $t$ test was used to assess the data, with $p \leq 0.05$ as significance criteria.

Immunohistochemistry. Control slices and slices treated with kinase inhibitors were immediately put in ice-cold $4 \%$ paraformaldehyde $/ 0.1 \%$ glutaraldehyde in PBS, pH 7.4, and fixed overnight. The slices were then put in $30 \%$ sucrose overnight at $4^{\circ} \mathrm{C}$ and embedded with optimal cutting temperature compound. The slices were sectioned into $20 \mu \mathrm{m}$ sections using a sliding microtome. Free-floating sections were blocked with $10 \%$ normal goat serum in PBS/0.7\% Triton X-100 (PBS-TX) overnight at $4^{\circ} \mathrm{C}$. Sections were then incubated overnight at $4^{\circ} \mathrm{C}$ with phospho- $4 \mathrm{E}-$ BP1 (Thr37/46) antibody (1:100). After washing three times with PBSTX, sections were incubated for $2 \mathrm{~h}$ at room temperature with Cy3conjugated AffiniPure goat anti-rabbit IgG diluted 1:500 in blocking solution. Sections were then washed and mounted onto poly-L-lysinecoated slides. Sections were analyzed and imaged using a Zeiss (Oberkochen, Germany) LSM 510 meta confocal microscope system.

Electrophysiological recordings. Extracellular recordings were obtained from area CA1 of the 400- $\mu \mathrm{m}$-thick slices described above. The slices were placed in an interface-recording chamber and equilibrated with oxygenated ACSF at a flow rate of $1 \mathrm{ml} / \mathrm{min}$ at $32^{\circ} \mathrm{C}$ for at least $1 \mathrm{~h}$ before recording. Extracellular recordings of field EPSPs (fEPSPs) were obtained from the stratum radiatum using microelectrodes filled with ACSF (resistance, 1-4 $\mathrm{M} \Omega$ ). A bipolar Teflon-coated platinum electrode was placed in the stratum radiatum to activate Schaffer collateral-commissural afferents at $0.05 \mathrm{~Hz}$. The stimulation strength was set to elicit a response equivalent to $50 \%$ of the maximal fEPSPs. In all experiments, baseline synaptic transmission was monitored for a minimum of $20 \mathrm{~min}$ before drug administration. The slope of the fEPSP was expressed as a percentage of the baseline average before drug application. Normalized data were averaged and expressed as the mean \pm SEM. Significant differences between groups were determined using two-way ANOVA with $p \leq 0.05$ as significance criteria.

\section{Results \\ Group I mGluRs couple to Mnk1 activation and eIF4E phosphorylation via ERK}

We demonstrated previously that the cap-dependent translation initiation pathway ERK2-Mnk1-eIF4E is conserved in mouse hippocampal area CA1 (Banko et al., 2004). Because inhibition of either ERK (Gallagher et al., 2004) or cap-dependent translation (Huber et al., 2000) blocks mGluR-LTD, we hypothesized that the activation of group I mGluRs should trigger activation of the ERK2-Mnk1-eIF4E signaling pathway. To test this hypothesis, we measured the levels of dually phosphorylated ERK2, phosphorylated Mnk1, and phosphorylated eIF4E after hippocampal slices were exposed to DHPG. A 5 min application of DHPG (50 $\mu \mathrm{M}$ ) induced maximal ERK2 activation with no overall effect on total ERK2 protein levels (Fig. 1A); thus, we used this treatment paradigm in subsequent experiments.

To identify the group I mGluR subtype responsible for the DHPG-induced activation of ERK2, we used two subtype-selective antagonists. LY367385 is a potent competitive antagonist that is selective for mGluR1, whereas MPEP is a potent noncompetitive antagonist that is selective for mGluR5. Interestingly, we found that both mGluR1 and mGluR5 were required to achieve maximal DHPG-induced ERK2 activation in hippocampal area CA1. Individual inhibition of mGluR1 or mGluR5 significantly attenuated the DHPG-induced activation of ERK2, whereas simultaneous inhibition of both subtypes completely blocked the DHPG-induced activation of ERK2 (Fig. 1B). Furthermore, DHPG-induced activation of ERK2 was preserved in synaptoneorosomes prepared post hoc, and the activation was blocked in both total homogenates and synaptoneurosomes by the mitogen-activated protein kinase kinase (MEK) inhibitor U0126 (Fig. 1C). These results demonstrate that group I mGluRs couple to the activation of ERK. 

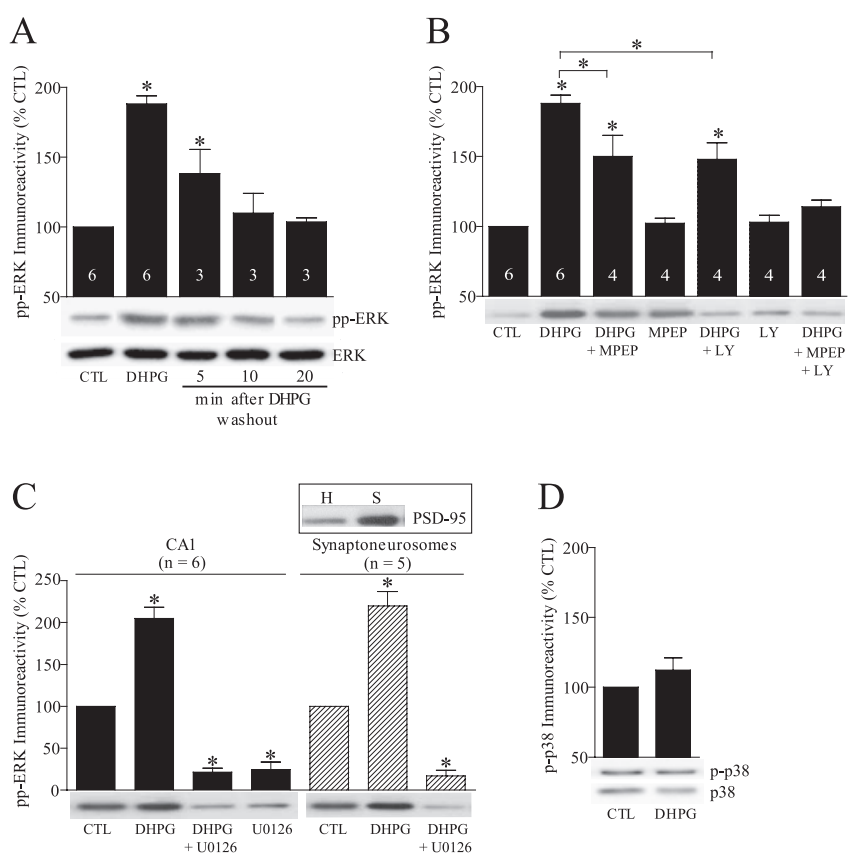

Figure 1. Activation of group I mGluRs results in activation of ERK. $\boldsymbol{A}, \mathrm{DHPG}$ application (50 $\mu \mathrm{m}, 5 \mathrm{~min}$ ) increases pp-ERK2 immunoreactivity. $\boldsymbol{B}$, Both mGluR1 (LY367385: $100 \mu \mathrm{m}, 20 \mathrm{~min}$ ) and mGluR5 (MPEP: $10 \mu \mathrm{m}, 20 \mathrm{~min}$ ) antaogonists inhibited maximum DHPG-induced ERK activation. C, DHPG-induced ERK activation was preserved in synaptoneurosomes and was blocked by preincubation with U0126 (20 $\mu \mathrm{m}, 30 \mathrm{~min}$ ). The inset shows PSD-95 (postsynaptic density-95) enrichment in the synaptoneurosome preparation ( $H$, total homogenate; $S$, synaptoneurosome). D, DHPG application did not result in an increase in $\mathrm{p} 38$ kinase phosphorylation $(n=4 ; p=0.23)$. Representative Western blots are shown in each panel. The cumulative data represent mean + SEM, and the number is indicated in the bars unless noted otherwise. ${ }^{*} p<$ 0.05 , statistical significance determined by Student's $t$ test. CTL, Control; LY, LY367385.

Several recent studies have provided information concerning the relative contributions of the ERK and p38 MAP kinase pathways to LTD. Electrically induced LTD ( $5 \mathrm{~Hz}, 3 \mathrm{~min}$ ) requires p38 and not ERK (Bolshakov et al., 2000), whereas DHPG-induced LTD appears to require ERK and not p38 (Gallagher et al., 2004). Therefore, we determined whether DHPG-induced activation of mGluRs results in activation of p38. In agreement with Gallager et al. (2004), we found that activation of the group I mGluRs via DHPG did not lead to an increase in the total p38 protein level or the dual phosphorylation of p38 at the residues required for kinase activity Thr180/Tyr182 (Fig. $1 D$ ). These results suggest that DHPG-induced mGluR-LTD does not elicit p38 activation.

DHPG application resulted in an increase in Mnk1 activation and eIF4E phosphorylation without affecting the total protein levels of either protein (Fig. $2 \mathrm{~A}$ ). These changes were preserved in synaptoneurosomes prepared post hoc and could be blocked by preincubation with U0126 (Fig. 2A). Consistent with our Western blot analysis of synaptoneurosomes, DHPG application also resulted in increased dually phosphorylated Mnk1 immunoreactivity in the dendritic layer of CA1 as well as in the soma when analyzed via immunohistochemistry (Fig. 2B). Notably, U0126 was ineffective at reducing basal phosphorylation levels of Mnk1 and eIF4E, suggesting that an alternative signaling pathway may operate to regulate Mnk1 and eIF4E in hippocampal slices under basal conditions. In addition to ERK, p38 has also been implicated in the upstream regulation of Mnk1 and eIF4E (Waskiewicz et al., 1997). Although we found no evidence for DHPG-induced p38 activation (Fig. 1D), or that the DHPG-induced increases in Mnk1 and eIF4E phosphorylation were p38 dependent with our
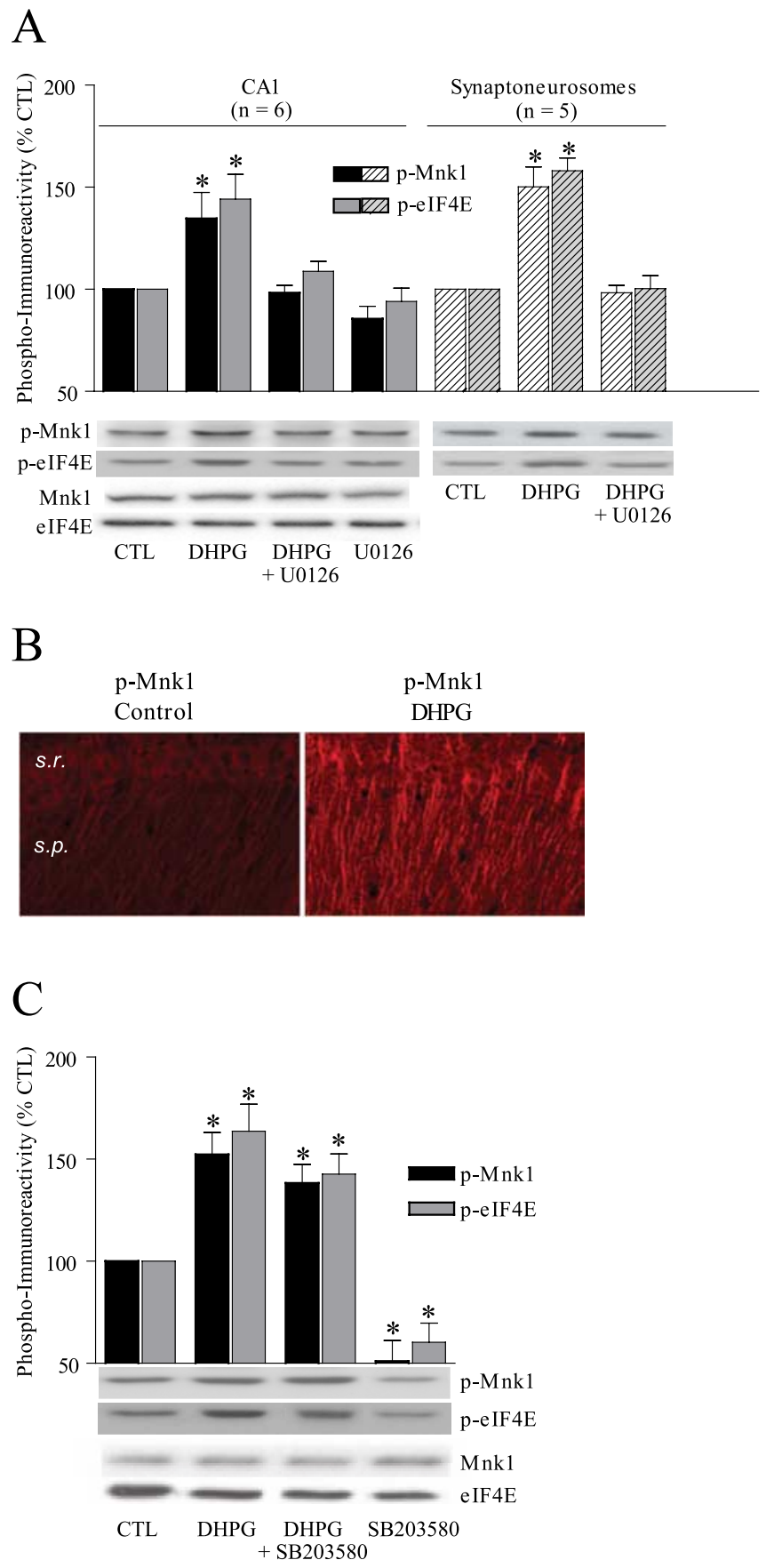

Figure 2. Activation of group I mGluRs results in Mnk1 activation and increased elF4E phosphorylation via ERK. A, DHPG-induced ( $50 \mu \mathrm{m}, 5 \mathrm{~min}$ ) increases in p-Mnk1 and p-elF4E immunoreactivity in both whole homogenates and synaptoneurosomes were ERK dependent (U0126: $20 \mu \mathrm{M}, 30 \mathrm{~min}$ ). $\boldsymbol{B}$, Representative micrograph of DHPG-induced increase in p-Mnk1 immunoreactivity in area CA1. s.p., Stratum pyramidal; s.r., stratum radiatum. C, Preincubation with p38 inhibitor SB203580 (1 $\mu \mathrm{M}, 1 \mathrm{~h})$ reduced basal Mnk1 and elF4E phosphorylation but did not significantly attenuate DHPG-induced Mnk1 activation and elF4E phosphorylation $(n=4)$. Representative Western blots are shown in each panel. The cumulative data represent mean + SEM. ${ }^{*} p<0.05$, statistical significance determined by Student's $t$ test. CTL, Control.

paradigm (Fig. 2C), inhibition of p38 activity with SB203580 significantly reduced basal phosphorylation levels of Mnk1 and eIF4E (Fig. 2C). Together, these data indicate that the group I mGluRs are coupled to cap-dependent translation initiation factors in the hippocampus and that their activation leads to the induction of an ERK2-Mnk1-eIF4E signaling pathway. 
A

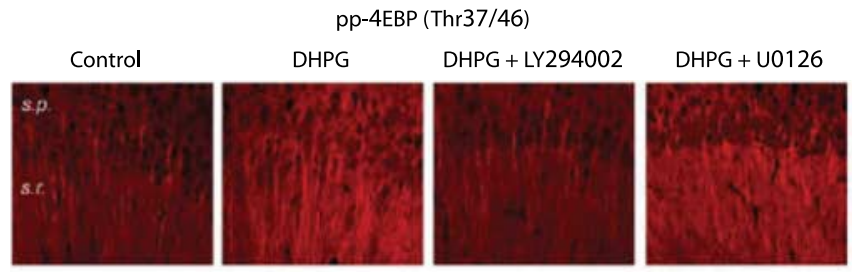

B

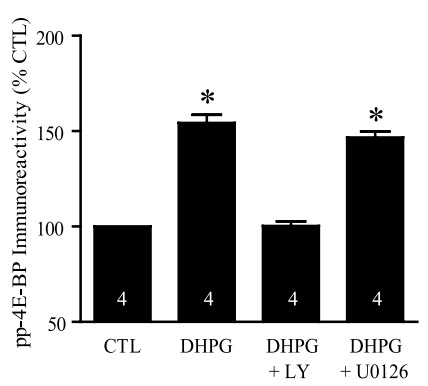

Figure 3. Activation of group I mGluRs results in increased 4E-BP phosphorylation via PI3K. $\boldsymbol{A}$, Representative micrographs demonstrating that DHPG application ( $50 \mu \mathrm{M}, 5 \mathrm{~min}$ ) resulted in an increase in pp-4E-BP immunoreactivity in area CA1. The DHPG-induced increase in 4E-BP phosphorylation was blocked by preincubation with LY294002 (50 $\mu \mathrm{m}, 30 \mathrm{~min}$ ) but not U0126 (20 $\mu \mathrm{m}, 30 \mathrm{~min})$ ( $n=4$ slices; 4 mice per condition). s.p., Stratum pyramidal; s.r., stratum radiatum. $\boldsymbol{B}$, Bar graph showing quantification of the immunocytochemical experiments in $\boldsymbol{A}$. The cumulative data represent mean $+S E M$, and the number is indicated in the bars. ${ }^{*} p<$ 0.05 , statistical significance determined by Student's $t$ test. CTL, Control; LY, LY294002.

\section{mGluR-LTD also recruits the PI3K-mTOR-4E-BP-eIF4E translation initiation pathway}

In addition to direct phosphorylation by Mnk1, eIF4E is regulated by sequestration via the $4 \mathrm{E}-\mathrm{BPs}$. Hyperphosphorylation of 4E-BP disrupts the 4E-BP-eIF4E inhibitory interaction. Because DHPG-induced LTD triggers the activation of the PI3K-mTOR pathway, we hypothesized that DHPG would also be effective at inducing 4E-BP phosphorylation. Indeed, we observed an increase in phosphorylated 4E-BP immunoreactivity in hippocampal area CA1 after DHPG application (Fig. 3). It should be noted that the phospho-specific 4E-BP1 antibody used cross-reacts with $4 \mathrm{E}-\mathrm{BP} 2$ and that $4 \mathrm{E}-\mathrm{BP} 2$ is the predominant $4 \mathrm{E}-\mathrm{BP}$ isoform expressed in the hippocampus. Because 4E-BP immunoreactivity is absent in 4E-BP2 knock-out animals (Banko et al., 2005), we conclude that the changes observed are specifically attributable to increased 4E-BP2 phosphorylation.

4E-BP2 phosphorylation is usually regulated via a PI3K-dependent pathway, involving signaling by the anti-apoptotic kinase Akt via mTOR (Raught and Gingras, 1999). Regulation of 4E-BP phosphorylation by ERK has also been demonstrated in hippocampal area CA1 (Kelleher et al., 2004). Because a role for both the PI3KAkt-mTOR signaling pathway (Hou and Klann, 2004) and ERK (Gallagher et al., 2004) in mGluR-LTD has been demonstrated, we next sought to determine which signaling pathway lies upstream of the DHPG-induced 4E-BP2 phosphorylation. Preincubation with the PI3K inhibitor LY294002, but not U0126, was effective at blocking the DHPG-induced increase in phosphorylated 4E-BP2 immunoreactivity (Fig. 3). These data suggest that during mGluR-LTD, the PI3K pathway couples the group I mGluRs to the regulation of 4E-BP2 phosphorylation at Thr37/46.
A

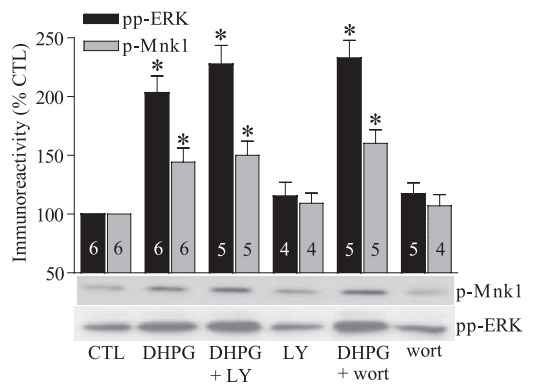

B
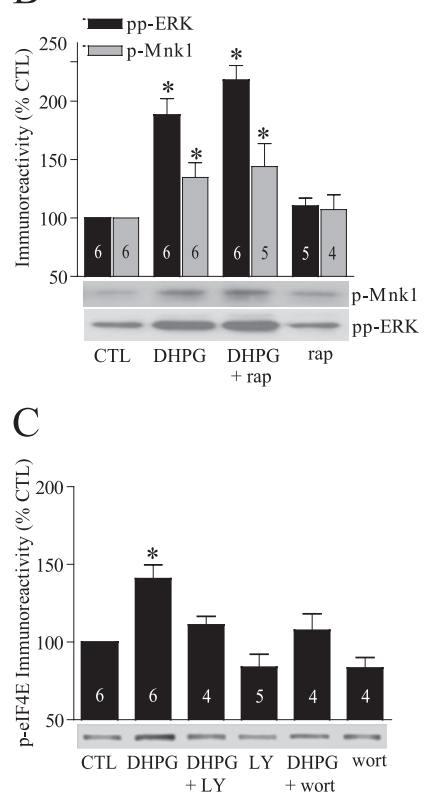

Figure 4. DHPG-induced regulation of elF4F requires PI3K/mTOR signaling. $A$, The PI3K inhibitors did not significantly alter the DHPG-induced activation of either ERK2 or Mnk1. $\boldsymbol{B}$, The mTOR inhibitor rapamycin (20 nm, $30 \mathrm{~min}$ ) did not significantly alter the DHPG-induced activation of either ERK2 or Mnk1. C, The PI3K inhibitors LY294002 (50 $\mu \mathrm{m}, 30 \mathrm{~min}$ ) and wortmannin (100 nm, $30 \mathrm{~min}$ ) blocked the DHPG-induced increase in elF4E phosphorylation. D, The mTOR inhibitor rapamycin $(20 \mathrm{~nm}, 30$ $\mathrm{min}$ ) blocked the DHPG-induced increase in elF4E phosphorylation. E, DHPG-induced increase in elF4E phosphorylation occurs in 4E-BP2 knock-out mice but is not sensitive to rapamycin ( $20 \mathrm{~nm}, 30 \mathrm{~min}$ ). Representative Western blots are shown in each panel. The cumulative data represent mean + SEM, and the number is indicated in the bars. ${ }^{*} p<0.05$, statistical significance determined by Student's $t$ test. CTL, Control; LY, LY294002; wort, wortmannin; rap, rapamycin.

\section{eIF4E integrates PI3K-mTOR and ERK signaling during mGluR-LTD}

Examples of cooperative PI3K and ERK cross talk have been demonstrated in hippocampal neurons. For example, PI3K activation is required for both NMDA- and amyloid- $\beta$ peptide-induced ERK activation (Opazo et al., 2003; Bell et al., 2004). Because our results suggest that both the PI3K and ERK pathways serve to couple group I mGluRs to translational machinery, we next examined potential cross talk between the two pathways after group I mGluR activation. Although there was a trend toward PI3K- and mTOR-dependent downregulation of ERK2, we were unable to observe a significant role for either PI3K or mTOR in the DHPG-induced activation of the ERK2-Mnk1 signaling pathway (Fig. 4A,B). When we examined the relationship between inhibition of either PI3K or mTOR on the DHPG-induced increased phosphorylation of eIF4E, however, we did observe a point of integration for these two signaling pathways. Preincubation with either the PI3K inhibitor LY294002 or the mTOR inhibitor rapamycin not only reduced the basal phosphory- 
A

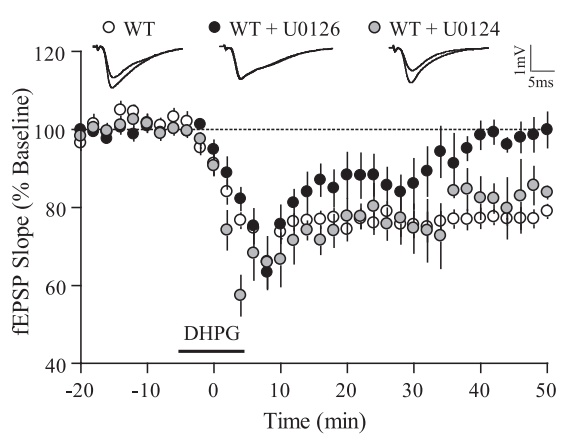

$\mathrm{C}$

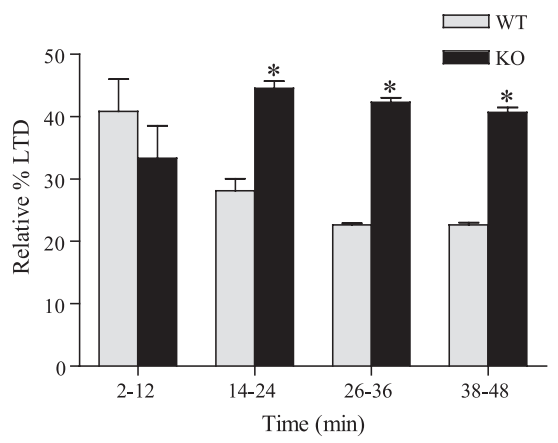

B

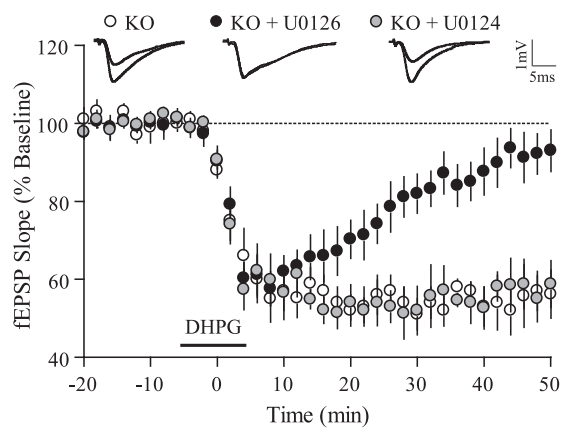

$\mathrm{D}$

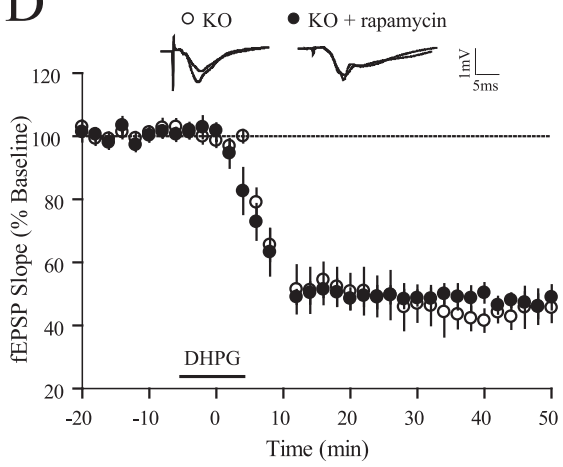

Figure 5. DHPG-induced mGluR-LTD is enhanced in 4E-BP2 knock-out mice. $A, D H P G$ application (50 $\mu \mathrm{M}, 10 \mathrm{~min}$ ) induced LTD in wild-type slices that was sensitive to preincubation with U0126 (20 $\mu \mathrm{m}, 1 \mathrm{~h}$ ) (ANOVA; $p<0.0001$ ) but not the inactive analog U0124 (20 $\mu \mathrm{M}, 1 \mathrm{~h}$ ) ( $n=5$ slices; 5 mice per condition). $B$, DHPG application (50 $\mu \mathrm{M}, 10 \mathrm{~min}$ ) induced LTD in 4E-BP2 knock-out slices that was sensitive to preincubation with U0126 (20 $\mu \mathrm{M}, 1 \mathrm{~h})$ (ANOVA; $p<0.0001)$ but not the inactive analog U0124 (20 $\mu \mathrm{M}, 1 \mathrm{~h})(n=5 \mathrm{slices} ; 5$ mice per condition). C, DHPG-induced LTD is enhanced in 4E-BP2 knock-out slices. The cumulative data are mean + SEM recapitulated from $\boldsymbol{A}$ and $\boldsymbol{B}$ for the indicated time periods. ${ }^{*} p<0.05$, statistical significance determined by Student's $t$ test. $\boldsymbol{D}, \mathrm{DHPG}$-induced LTD in 4E-BP2 knock-out mice is insensitive to rapamycin ( $n=4$ slices; 4 mice per condition). WT, wild type; K0, knock-out.

lation of eIF4E but also attenuated the DHPG-induced increase in eIF4E phosphorylation (Fig. 4C,D). Direct phosphorylation of eIF4E at Ser209 by mTOR has not been reported. It is more likely that 4E-BP2 mediates the observed dependence of eIF4E phosphorylation on PI3K-mTOR signaling. Consistent with this notion, DHPG-induced eIF4E phosphorylation is insensitive to rapamycin in 4E-BP2 knock-out slices (Fig. 4E). Together with the aforementioned requirement for MEK-ERK signaling to achieve DHPGinduced eIF4E phosphorylation, these results suggest that the concomitant activation of both the PI3K-mTOR and ERK signaling pathways converge to regulate eIF4E phosphorylation during mGluR-LTD.

\section{mGluR-LTD is enhanced in mice that lack 4E-BP2}

Gallagher et al. (2004) have demonstrated that inhibition of the MEK-ERK signaling pathway blocks the maintenance of mGluRLTD. In complete agreement with their report, we found that application of the MEK inhibitor U0126 blocked long-lasting DHPGinduced LTD, whereas the inactive analog U0124 did not (Fig. 5A). In studies described previously, we demonstrated that one of the functions of the DHPG-induced activation of ERK is to engage the cap-dependent translation initiation factor eIF4E. In an effort to determine the extent to which the regulation of eIF4E impacts mGluR-LTD, we investigated DHPG-induced LTD in mice engineered to lack 4E-BP2. We recently characterized NMDA receptordependent long-term potentiation (LTP) in hippocampal area CA1 and determined that basal synaptic transmission is unaltered in the
4E-BP2 knock-out mice (Banko et al., 2005). When exposed to the same DHPG treatment as wild-type hippocampal slices, the 4E-BP2 knock-out slices exhibited a more robust LTD (Fig. $5 B, C$ ). The mechanism for the enhanced LTD in the 4E-BP2 knock-out mice requires ERK activation, because preincubation with U0126 blocked completely the DHPG-induced LTD (Fig. 5B). These results demonstrate that ERK-dependent regulation of eIF4E contributes to mGluR-LTD.

It was demonstrated previously that mGluR-LTD induced by DHPG is sensitive to inhibition of mTOR activity with rapamycin (Hou and Klann, 2004). Strikingly, the LTD exhibited by the 4E-BP2 knock-out animals is not sensitive to mTOR inhibition by rapamycin (Fig. 5D). These results are surprising because $4 \mathrm{E}$ BP2 is just one of several downstream effector molecules of mTOR thought to be critical for protein synthesis (Klann and Dever, 2004; Klann et al., 2004) and suggest that, under normal circumstances, the most significant consequence of mTOR activation during mGluR-LTD (Hou and Klann, 2004) is the regulation of 4E-BP2.

ERK-dependent Mnk1 phosphorylation of eIF4E can only occur when eIF4E is bound to eIF4G in the eIF4F complex (Pyronnet et al., 1999). Consistent with the hypothesis that 4E-BP2 serves to sequester eIF4E away from eIF4G, we found that basal levels of eIF4F complex are elevated in the 4E-BP2 knock-out mice (Fig. 6) (Banko et al., 2005). Because mGluR-LTD is enhanced in the $4 \mathrm{E}$ BP2 knock-out mice, it was pertinent to examine eIF4F complex formation during mGluR-LTD in the 4E-BP2 knock-out mice. Our previous report indicates that total eIF4E protein levels do not differ between wild-type and 4E-BP2 knock-out animals (Banko et al., 2005), therefore any changes observed in eIF4F complex levels between wild-type and knock-out animals is attributable to the lack of 4E-BP2 rather than the abundance of eIF4E. In wild-type mice, DHPG-induced LTD was associated with an increase in eIF4F complex levels $10 \mathrm{~min}$ after LTD induction (Fig. 6). This result is consistent with the hypothesis that cap-dependent translation is engaged during mGluR-LTD. Notably, we did not observe an additional increase in eIF4F complex levels after LTD induction in the 4E-BP2 knock-out slices. In addition, we found rapamycin inhibited DHPGinduced eIF4F complex formation in slices from wild-type mice but not in slices from 4E-BP2 knock-out mice (Fig. 6). This finding is consistent with our electrophysiological studies that show that rapamycin inhibits mGluR-LTD in wild-type mice (Hou and Klann, 2004) but has no effect on mGluR-LTD in 4E-BP2 knock-out mice (Fig. 5D). These observations implicate 4E-BP2 as the prominent eIF4E-binding protein that is engaged during mGluR-LTD. This is in contrast to LTP. We demonstrated previously that LTP-inducing stimulation results in a greater abundance of the eIF4F complex in both wild-type and 4E-BP2 knock-out slices (Banko et al., 2005). Together, these results directly demonstrate that cap-dependent translation initiation is engaged during mGluR-LTD and that 4E$\mathrm{BP} 2$ regulates eIF4E availability for eIF4F initiation complex assembly. 


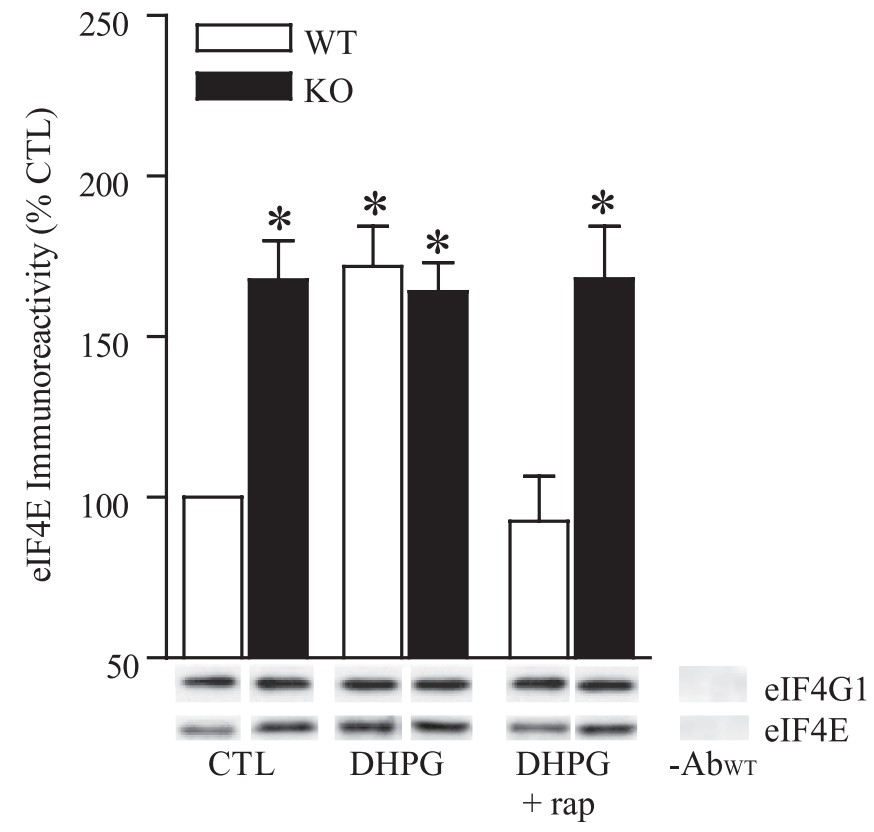

Figure 6. elF4F complex formation is upregulated during DHPG-induced mGluR-LTD. Basal coimmunoprecipitation of elF4E with elF4G was elevated in 4E-BP2 knock-out (KO) slices ( $n=$ 7 slices; 7 mice per genotype). mGluR-LTD is associated with increased coimmunoprecipitation of elF4E with elF4G in wild-type (WT) slices but not in 4E-BP2 knock-out slices 10 min after DHPG application ( $n=7$ slices; 7 mice per genotype). Rapamycin (rap) inhibited the DHPG-induced elF4G-elF4E association in wild-type but not in 4E-BP2 knock-out slices ( $n=3$ slices; 3 mice per genotype). Representative elF4G and elF4E Western blots are shown for each condition along with bead alone $\left(-A b_{W T}\right)$ control. The cumulative data represent mean + SEM. ${ }^{*} p<$ 0.05 , statistical significance determined by Student's $t$ test. CTL, Control.

\section{Discussion}

\section{Cap-dependent translation contributes to mGluR-LTD}

De novo mRNA translation is essential for the expression of DHPG-induced mGluR-LTD (Huber et al., 2000, 2001). mRNA translation is often grouped into distinct classes based on regulatory elements inherent to the different mRNA families. For example, 5' TOP-mediated translation occurs when the mRNA contains a polypyrimidine tract in the $5^{\prime}$ noncoding region termed the 5' TOP sequence. Internal ribosome entry site (IRES)-mediated translation occurs when the mRNA contains an IRES, and cap-dependent translation occurs when binding of the eIF4F complex to the $5^{\prime}$ cap structure is required for ribosome association. Cap-dependent translation plays a critical role during mGluR-LTD because addition of the exogenous cap analog is sufficient to block the maintenance of mGluR-LTD (Huber et al., 2000). In this study, we directly demonstrated that capdependent translation is engaged during DHPG-induced mGluR-LTD by demonstrating an increase in eIF4F complex formation after induction of mGluR-LTD (Fig. 6). Moreover, our data suggest that further increasing the levels of the eIF4F complex available to initiate cap-dependent translation by the genetic elimination of 4E-BP2 results in an enhancement in mGluR-LTD induced by DHPG (Fig. 5). The observation that the enhanced LTD exhibited by the 4E-BP2 knock-out mice was not associated with a further increase in eIF4F complex levels is in sharp contrast to what we have observed in LTP studies (Banko et al., 2005). Importantly, this observation depicts a fundamental difference in the translation initiation regulatory mechanisms that belie LTP and LTD. Although both forms of plasticity are dependent on cap-dependent translation, the availability of eIF4E to form the eIF4F initiation complex appears to be primarily regulated by
4E-BP2 during mGluR-LTD; however, the availability of eIF4E to form the eIF4F initiation complex is likely regulated by other eIF4E-binding proteins in addition to 4E-BP2 during LTP (Banko et al., 2005).

\section{The ERK and PI3K signaling pathways couple group I mGluRs to cap-dependent translation initiation factors} Previous studies have shown that DHPG-induced mGluR-LTD is dependent on MEK-ERK signaling (Gallagher et al., 2004) and PI3K-Akt-mTOR signaling (Hou and Klann, 2004). One role these pathways may serve during mGluR-LTD is to engage translation factors. In this study, we have shown that activation of group I mGluRs with DHPG can trigger the activation of an ERK2-Mnk1-eIF4E signaling pathway (Figs. 1,2) as well as a PI3K-mTOR-4E-BP-eIF4E (Figs. 3, 4) signaling pathway in mouse hippocampal area CA1. Consistent with the notion that DHPG can induce rapid local protein synthesis (Weiler and Greenough, 1993; Huber et al., 2000; Job and Eberwine, 2001), several of the DHPG-induced changes in phosphorylation of the translation factors we investigated were preserved in synaptoneurosome preparations and could be observed in the dendrites of area CA1.

It is apparent that both the ERK-MEK and PI3K-Akt-mTOR pathways are activated concomitantly after group I mGluR activation and that they each signal to cap-dependent translation initiation factors, but we could not indisputably demonstrate a cooperative cross talk between the two (Fig. 4). We propose that eIF4E serves as the point of signal integration for the convergence of the two pathways (Fig. 4). Our current findings coupled with our previous studies (Hou and Klann, 2004) indicate that DHPGinduced activation of the group I mGluRs engages the PI3K-AktmTOR pathway, resulting in phosphorylation of 4E-BP2, thereby relieving the inhibition of eIF4E. This relief of inhibition permits eIF4E to join to eIF4G and form the eIF4F initiation complex, which initiates translation. Once bound to eIF4G, eIF4E can be phosphorylated by Mnk1, a process that strongly correlates with increased mRNA translation (Gingras et al., 1999).

Another MAP kinase pathway that has been shown to activate Mnk1 and regulate eIF4E phosphorylation after stress stimuli is the p38 pathway (Fukunaga and Hunter, 1997; Waskiewicz et al., 1997). It has been reported that p38 kinase plays a role during mGluR-LTD (Bolshakov et al., 2000; Huang et al., 2004). However, in agreement with Gallagher et al. (2004), we found no evidence for DHPG-induced p38 activation using our LTDinduction paradigm (Fig. 1). Moreover, DHPG-induced Mnk1 activation and increase in eIF4E phosphorylation was not blocked by inhibition of p38 (Fig. 2). It is noteworthy that the p38 inhibitor did reduce the basal phosphorylation levels of Mnk1 and eIF4E in whole homogenates of hippocampal area CA1, whereas the ERK inhibitor did not. These data implicate ERK as a selective upstream regulator of Mnk1-eIF4E signaling that is activated in response to synaptic stimulation but not a prominent regulator of Mnk1-eIF4E signaling under basal conditions. Consistent with this notion, we also found that NMDA receptor activation resulted in a similar pattern of activation of the ERK2Mnk1-eIF4E pathway (Banko et al., 2004). Together, these data suggest the intriguing possibility that in hippocampal area CA1, the p38-Mnk1-eIF4E pathway operates on a global level to maintain basal translation, whereas the ERK2-Mnk1-eIF4E pathway is selectively engaged during periods of synaptic activity. 


\section{A possible mechanism to identify the synaptic plasticity proteins}

Long-term memory and enduring forms of synaptic plasticity are distinguished from their short-lasting counterparts by their dependence on protein synthesis. We and others have recently begun to define the signaling mechanisms coupling synaptic activation to the protein synthesis machinery. An important question that has not been investigated to date on a broad scale is the identity of the newly synthesized proteins. This is likely because experiments such as those described in this study that are aimed to identify which categories of mRNA translation are engaged during the aforementioned processes are only beginning to be undertaken. As the translation regulatory mechanisms that contribute to synaptic plasticity are revealed through the use of pharmacological agents and mutant mice, investigators will be better poised to address the difficult but critical question of the identity of the mRNAs being translated during various forms of translation-dependent synaptic plasticity.

Two mutant mice that hold promise for such experiments are mice that model Fragile $\mathrm{X}$ mental retardation and the $4 \mathrm{E}$ BP2 knock-out mice used here. Huber et al. (2002) have shown that hippocampal mGluR-LTD is enhanced in slices from mice that lack Fragile $\mathrm{X}$ mental retardation protein (FMRP), suggesting an important role for FMRP in translational control during mGluR-LTD. In this study, we demonstrated that mGluR-LTD is also enhanced in 4E-BP2 knockout mice (Fig. 5). Additionally, it is also altered in the 4E-BP2 knock-out mice (Banko et al., 2005). Examination of the altered protein compositions in these mice after stimulation that triggers synaptic plasticity should provide insight into the identity of the specific proteins translated in response to mGluR-LTD and LTP.

\section{References}

Banko JL, Hou L, Klann E (2004) NMDA receptor activation results in PKA- and ERK-dependent Mnk1 activation and increased eIF4E phosphorylation in hippocampal area CA1. J Neurochem 91:462-470.

Banko JL, Poulin F, Hou L, DeMaria C, Sonenberg N, Klann E (2005) The translation repressor 4E-BP2 is critical for eIF4F complex regulation, synaptic plasticity and memory in the hippocampus. J Neurosci 25:9581-9590.

Bell KA, O’Riordan KJ, Sweatt JD, Dineley KT (2004) MAPK recruitment by beta-amyloid in organotypic hippocampal slice cultures depends on physical state and exposure time. J Neurochem 91:349-361.

Beretta L, Gingras AC, Svitkin YV, Hall MN, Sonenberg N (1996) Rapamycin blocks the phosphorylation of 4E-BP1 and inhibits cap-dependent initiation of translation. EMBO J 15:658-664.

Bolshakov VY, Siegelbaum SA (1994) Postsynaptic induction and presynaptic expression of hippocampal long-term depression. Science 264:1148-1152.

Bolshakov VY, Carboni L, Cobb MH, Siegelbaum SA, Belardetti F (2000) Dual MAP kinase pathways mediate opposing forms of long-term plasticity at CA3-CA1 synapses. Nat Neurosci 3:1107-1112.

Bradford MM (1976) Rapid and sensitive method for quantification of microgram quantities of protein using the principle of protein-dye binding. Anal Biochem 72:248-252.

Fitzjohn SM, Kingston AE, Lodge D, Collingridge GL (1999) DHPGinduced LTD in area CA1 of juvenile rat hippocampus; characterisation and sensitivity to novel mGlu receptor antagonists. Neuropharmacology 38:1577-1583.

Fukunaga R, Hunter T (1997) MNK1, a new MAP kinase-activated protein kinase, isolated by a novel expression screening method for identifying protein kinase substrates. EMBO J 16:1921-1933.

Gallagher SM, Daly CA, Bear MF, Huber KM (2004) Extracellular signalregulated protein kinase activation is required for metabotropic glutamate receptor-dependent long-term depression in hippocampal area CA1. J Neurosci 24:4859-4864.

Gingras AC, Sonenberg N (1997) Adenovirus infection inactivates the translational inhibitors 4E-BP1 and 4E-BP2. Virology 237:182-186.
Gingras AC, Kennedy SG, O’Leary MA, Sonenberg N, Hay N (1998) 4E$\mathrm{BP} 1$, a repressor of mRNA translation, is phosphorylated and inactivated by the Akt(PKB) signaling pathway. Genes Dev 12:502-513.

Gingras AC, Raught B, Sonenberg N (1999) eIF4 initiation factors: effectors of mRNA recruitment to ribosomes and regulators of translation. Annu Rev Biochem 68:913-963.

Hay N, Sonenberg N (2004) Upstream and downstream of mTOR. Genes Dev 18:1926-1945.

Hou L, Klann E (2004) Activation of the phosphoinositide 3-kinase-Aktmammalian target of rapamycin signaling pathway is required for metabotropic glutamate receptor-dependent long-term depression. J Neurosci 24:6352-6361.

Huang CC, You JL, Wu MY, Hsu KS (2004) Rap1-induced p38 mitogenactivated protein kinase activation facilitates AMPA receptor trafficking via the GDI.Rab5 complex. Potential role in ( $S$-3,5-dihydroxyphenylglyceneinduced long term depression. J Biol Chem 279:12286-12292.

Huber KM, Kayser MS, Bear MF (2000) Role for rapid dendritic protein synthesis in hippocampal mGluR-dependent long-term depression. Science 288:1254-1257.

Huber KM, Roder JC, Bear MF (2001) Chemical induction of mGluR5- and protein synthesis-dependent long-term depression in hippocampal area CA1. J Neurophysiol 86:321-325.

Huber KM, Gallagher SM, Warren ST, Bear MF (2002) Altered synaptic plasticity in a mouse model of fragile $\mathrm{X}$ mental retardation. Proc Natl Acad Sci USA 99:7746-7750.

Job C, Eberwine J (2001) Identification of sites for exponential translation in living dendrites. Proc Natl Acad Sci USA 98:13037-13042.

Kelleher III RJ, Govindarajan A, Jung HY, Kang H, Tonegawa S (2004) Translational control by MAPK signaling in long-term synaptic plasticity and memory. Cell 116:467-479.

Klann E, Dever TD (2004) Biochemical mechanisms for translational regulation in synaptic plasticity. Nat Rev Neurosci 5:931-942.

Klann E, Antion MD, Banko JL, Hou L (2004) Synaptic plasticity and translation initiation. Learn Mem 11:365-372.

Lin TA, Lawrence Jr JC (1996) Control of the translational regulators PHAS-I and PHAS-II by insulin and cAMP in 3T3-L1 adipocytes. J Biol Chem 271:30199-30204.

Mulkey RM, Malenka RC (1992) Mechanisms underlying induction of homosynaptic long-term depression in area CA1 of the hippocampus. Neuron 9:967-975.

Oliet SH, Malenka RC, Nicoll RA (1997) Two distinct forms of long-term depression coexist in CA1 hippocampal pyramidal cells. Neuron 18:969-982.

Opazo P, Watabe AM, Grant SG, O’Dell TJ (2003) Phosphatidylinositol 3-kinase regulates the induction of long-term potentiation through extracellular signal-related kinase-independent mechanisms. J Neurosci 23:3679-3688.

Palmer MJ, Irving AJ, Seabrook GR, Jane DE, Collingridge GL (1997) The group I mGlu receptor agonist DHPG induces a novel form of LTD in the CA1 region of the hippocampus. Neuropharmacology 36:1517-1532.

Pause A, Belsham GJ, Gingras AC, Donze O, Lin TA, Lawrence Jr JC, Sonenberg N (1994) Insulin-dependent stimulation of protein synthesis by phosphorylation of a regulator of $5^{\prime}$-cap function. Nature 371:762-767.

Poulin F, Gingras AC, Olsen H, Chevalier S, Sonenberg N (1998) 4E-BP3, a new member of the eukaryotic initiation factor 4E-binding protein family. J Biol Chem 273:14002-14007.

Pyronnet S, Imataka H, Gingras AC, Fukunaga R, Hunter T, Sonenberg N (1999) Human eukaryotic translation initiation factor 4G (eIF4G) recruits mnk1 to phosphorylate eIF4E. EMBO J 18:270-279.

Raught B, Gingras AC (1999) eIF4E activity is regulated at multiple levels. Int J Biochem Cell Biol 31:43-57.

Snyder EM, Philpot BD, Huber KM, Dong X, Fallon JR, Bear MF (2001) Internalization of ionotropic glutamate receptors in response to mGluR activation. Nat Neurosci 4:1079-1085.

Vanderklish PW, Edelman GM (2002) Dendritic spines elongate after stimulation of group 1 metabotropic glutamate receptors in cultured hippocampal neurons. Proc Natl Acad Sci USA 99:1639-1644.

Waskiewicz AJ, Flynn A, Proud CG, Cooper JA (1997) Mitogen-activated protein kinases activate the serine/threonine kinases Mnk1 and Mnk2. EMBO J 16:1909-1920.

Weiler IJ, Greenough WT (1993) Metabotropic glutamate receptors trigger postsynaptic protein synthesis. Proc Natl Acad Sci USA 90:7168-7171. 